\title{
Radiologists Detect A Higher Frequency of Incidental Findings On Radiotherapy-Planning CT Scans of Breast Cancer Patients Than Oncologist
}

Stine Nyby ( $\nabla$ stinenyby@live.dk )

Aalborg University https://orcid.org/0000-0001-6131-8934

Signe Hertz Hansen

Aalborg University

Sophie Yammeni

Aalborg University Hospital

Agnieszka Monika Delekta

Aalborg University Hospital

Gintare Naujokaite

Aalborg University Hospital

Ursula G. Falkmer

Aalborg University

Martin Bøgsted

Aalborg University

Andreas Carus

Aalborg University

\section{Research Article}

Keywords: Breast cancer, Incidental findings, Interobserver variability, Planning CT scans, Radiotherapy

Posted Date: January 3rd, 2022

DOI: https://doi.org/10.21203/rs.3.rs-1214703/v1

License: (1) (1) This work is licensed under a Creative Commons Attribution 4.0 International License.

Read Full License 


\section{Abstract}

Purpose: Breast cancer patients scheduled for postoperative radiotherapy undergo radiotherapy-planning computed tomography (CT), and incidental findings (IFs) may appear. This study investigated the interobserver variability between radiologists and oncologists when assessing IFs on radiotherapyplanning CT scans in breast cancer patients prior to adjuvant radiotherapy.

Methods: We included 383 breast cancer patients who underwent planning CT at the Aalborg University Hospital between February 1, 2017 and February 28, 2018. IFs noted by the oncologists were identified from medical records. Two specialized radiologists reviewed the scans and described their IFs. IFs were classified as benign or potential malignant lesions. Cohen's kappa statistic was used to measure interobserver agreement.

Results: A total of 513 IFs were registered. The radiologists registered 433 findings, and the oncologists noted 80 (1.1 and 0.2 IFs per patient, respectively). Most potential malignant IFs were found in the liver, lungs, bones, and lymph nodes. The radiologists and oncologists detected potential malignant lesions in 94 (25\%) and 34 (9\%) patients, respectively. The oncologists' sensitivity for detecting IFs in the liver and lungs were $29 \%$ and $20 \%$, respectively. The agreements on IFs in the liver and lungs were fair (Cohen's kappa values of 0.33 and 0.28 , respectively).

Conclusion: Radiologists reported a significantly higher frequency of IFs and potential malignant lesions than oncologists. Additionally, the oncologists had a low sensitivity when reporting IFs in both the liver and lungs. These results emphasize the need for specialized radiologists to scrutinize planning CT scans of breast cancer patients to ensure the intention to treat.

\section{Introduction}

More than 5000 Danish women are diagnosed with breast cancer (BC) each year [1], and $85-90 \%$ are diagnosed at an early stage [2]. Early BC is locally staged with mammography and ultrasonography, and diagnostic imaging modalities such as computed tomography (CT) are not routinely performed because asymptomatic distant metastasis is rare [2,3]. Selected patients with locally advanced BC may undergo further diagnostic imaging, such as magnetic resonance imaging or positron emission tomography $\mathrm{CT}$, depending on risk factors and clinical assessment [3].

Modern BC treatment consists of surgery, postoperative radiation therapy, and systemic treatment. Seventy percent of BC patients who undergo surgery received breast-conserving surgery (lumpectomy), and $30 \%$ undergo mastectomy. Patients who undergo lumpectomy for ductal carcinoma in situ or invasive cancer without macrometastasis to the regional lymph nodes are typically offered postoperative radiotherapy [4].

Patients scheduled for postoperative radiotherapy undergo a radiotherapy-planning CT scan of the thorax and upper abdomen [5], and a dose plan is designed to determine the radiation dose distribution and 
minimize exposure to vital organs, such as the heart and lungs [6]. Even though the planning CT scan is not a diagnostic scan, incidental findings (IFs) may appear.

An IF is a finding that is not directly related to the purpose of the scan [7]. IFs can be either benign or potential malignant lesions, such as metastasis or synchronous cancer. The occurrence of IFs may have a clinically significant impact on the treatment course [6, 8-12]. However, there is a lack of information concerning the optimal review of these scans in relation to IFs when postoperative radiotherapy is planned. Radiologists are not routinely involved in daily clinical practice at our hospital; therefore, oncologists solely review the planning CT scans. The oncologists may not have any formal radiology education, and thus may have varying radiologic qualifications. In this study, we aimed to investigate the frequency of IFs detected by radiologists and oncologists to evaluate the assessment of planning CT scans.

\section{Methods}

We retrospectively identified planning CT scans of $439 \mathrm{BC}$ patients who were postoperatively treated with radiotherapy at the Department of Oncology, Aalborg University Hospital, Denmark between February 1 , 2017 and February 28, 2018. Of these, 383 patients who underwent radical surgery were included in the study (Figure 1).

\section{Planning CT scans}

The planning CT scans were performed with a Siemens SOMATOM go.Open Pro scanner (Siemens Healthcare $\mathrm{GmbH}$, Erlangen, Germany) with a slice thickness of $2.5 \mathrm{~mm}$ and without contrast agent. Patients were placed in the supine position, and a baseplate was used for the arms and head (Posirest ${ }^{\mathrm{TM}}$ 2). The arm of the affected side was placed above the head, and the opposite arm was placed alongside the body. For guidance, the mammary glands and/or cicatrice were marked with copper thread. The radiographer controlled the location of the reference point and the inclusion of the lungs on the "scout" image. The lungs and heart were always included on the CT scan, but the extent of the abdominal structures differed.

\section{Incidental findings}

An IF was defined as a radiological abnormality found on a planning CT scan. To establish the frequency of IFs registered by the oncologists, the patients' medical records were scrutinized, and any noted IF was recorded. Two specialized radiologists blinded to the patients' medical records or clinical information reviewed the planning CT scans. The radiologists' descriptions were considered the standard in terms of detecting IFs due to their specialization in assessing diagnostic images. Radiologic abnormalities on described prior diagnostic imaging were not considered when registering IFs.

IFs were categorized according to their anatomical location and classified as benign or potential malignant lesions. A potential malignant lesion was defined in a joint consultation between the 
oncologist and the radiologist, and additional examinations, such as ultrasonography or diagnostic CT, were performed. A benign IF was defined as an IF not requiring further examination.

\section{Data collection}

Demographic data and clinical characteristics were collected, including age at planning CT scan, sex, tumor size, lymph node status, histopathology, type of surgery, and other oncological treatments. All data were managed and stored in the Research Electronic Data Capture (REDCap®, version 9.5.6, Vanderbilt University, USA) hosted at the Aalborg University Hospital, Denmark.

\section{Statistical analysis}

Descriptive statistics were used to summarize demographic data and clinical characteristics. Two by two tables were used to compare the IFs observed by radiologists and oncologists. The sensitivity, specificity, positive predictive value (PPV), negative predictive value (NPV) and false positive were calculated. Cohen's kappa statistics (kappa) were used to quantify the agreement between the oncologists and radiologists. Kappa was calculated to determine the difference between the observed agreement and the agreement that could be expected by chance. The kappa statistic ranges from -1 to 1 , where 1 represents a perfect agreement and 0 equates agreement by chance. Negative values imply disagreement between the observers [14]. All data were analyzed using Stata ${ }^{\circledR}$ (Stata/MP 16.0, StataCorp LLC, Texas, USA).

\section{Results}

We included 383 patients, and their characteristics are summarized in Table 1. The median age was 61 years (26-86 years). Most patients had pT1 disease (65.5\%).

Table 1: Clinical characteristics of the 383 patients 
Age at CT scan, years, median (range)

$61(26-86)$

Sex (female)

$382(99)$

\section{Tumor size}

pT1 (£20 mm)

pT2 (20-50 mm)

pT3 (>50 mm)

pT4 (Any size /direct extension ${ }^{\mathrm{a}}$ )

Unknown primary tumor

Carcinoma in situ (ductal or lobular)

\section{Lymph nodes}

pNO (No regional lymph node metastasis)

pN1 (1-3 axillary lymph nodes)

pN2 (4-9 axillary lymph nodes)

pN3 ( ${ }^{3} 10$ axillary lymph nodes or $\left.{ }^{b}\right)$

Carcinoma in situ (ductal or lobular)

Histopathological classification

Carcinoma in situ (ductal or lobular)

Invasive ductal carcinoma

Invasive lobular carcinoma

Mixed invasive lobular and ductal carcinoma

Other malignant tumors ${ }^{\mathrm{c}}$

\section{Type of surgery}

Lumpectomy

Mastectomy

No surgery ${ }^{d}$

Sentinel node (SN)

Axillary lymph node dissection

Neither SN nor node dissection
$251(66)$

$98(26)$

$11(3)$

$2(0.5)$

$4(1)$

$17(4)$

$206(54)$

$125(33)$

$22(6)$

$13(3)$

$17(4)$

$17(4)$

287 (75)

44 (12)

10 (3)

25 (7)

309 (81)

70 (18)

4 (1)

237 (62)

133 (35)

$13(3)$ 
Data are presented as $\mathrm{n}(\%)$ unless otherwise indicated.

${ }^{\mathrm{a}}$ Extension to the chest wall or skin.

${ }^{\mathrm{b}}$ Axillary, infraclavicular, or ipsilateral supraclavicular lymph nodes.

'Mucinous adenocarcinoma $(n=11)$, metaplastic carcinoma $(n=2)$, apocrin carcinoma $(n=1)$, invasive solid papillary carcinoma $(n=5)$, tubular carcinoma $(n=2)$, adenocarcinoma $(n=2)$, secretory carcinoma $(n=1)$, and intracystic carcinoma $(n=1)$.

${ }^{\mathrm{d} D u e}$ to unknown primary tumor.

\section{Organ distribution of incidental findings}

A total of 513 IFs were detected: 433 by the radiologists and 80 by the oncologists. The radiologist and oncologists detected an average of 1.1 and 0.2 IFs per patient, respectively. The radiologists detected potential malignant lesions in 94 patients (25\%), whereas the oncologists found potential malignant lesions in only 34 patients (9\%). By contrast, the radiologists detected benign IFs in 162 (42\%) patients, whereas the oncologists noted benign IFs in 39 patients (10\%).

The breast, liver, and lungs were the most frequent locations of IFs. The distribution of IFs by organ is shown in Table 2. If a patient presented with several IFs, each IF was registered according to its anatomic location, independent of the individual patient. The breast was the most common site of benign findings registered by the radiologists ( $83 \mathrm{IFs}$ ), whereas the liver was the most common site of benign findings detected by the oncologists ( $16 \mathrm{IFs}$ ). The liver was the most frequent site of potential malignant lesions; the radiologists reported $41 \mathrm{IFs}$ in the liver, whereas the oncologists noted 19. The majority of potential malignant lesions were located in the liver and lungs.

Table 2: Distribution of incidental findings registered by the radiologists and oncologists 


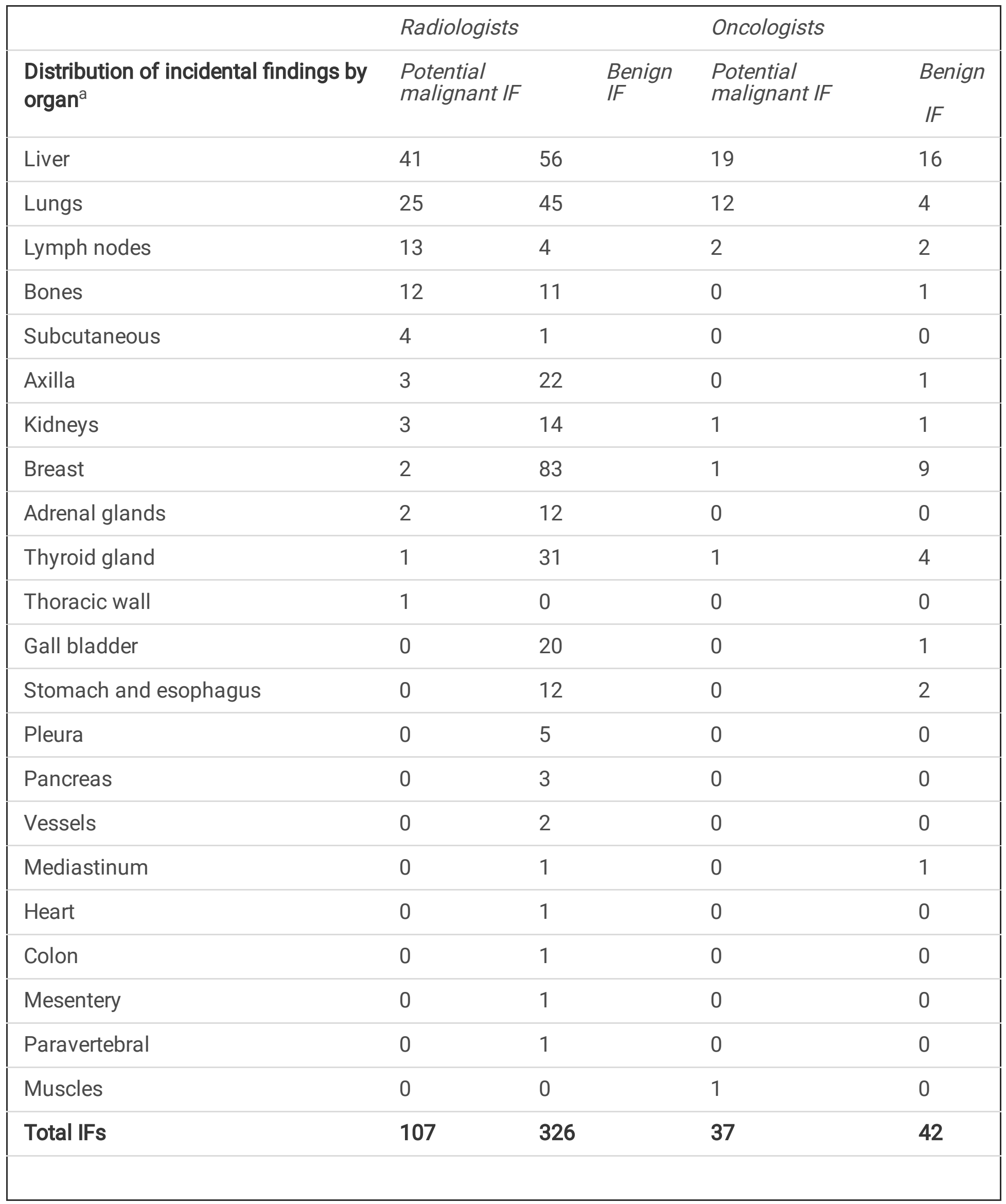

aSome patients had more than one incidental finding on the computed tomography scan. 
IF: incidental finding

\section{Interobserver variability}

The associations between the potential malignant lesions detected by radiologists and oncologists are shown in Table 3. Of the 383 included patients, the radiologists registered 97 patients with IFs in the liver and 70 with IFs in the lungs. By contrast, the oncologists reported 35 patients with IFs in the liver and 16 with IFs in the lungs.

Table 3: Two by two tables summarizing the number of patients with and without incidental findings in the $A$ ) liver and $B$ ) lungs registered by the oncologists and the radiologists

1. Liver

Radiologists

\begin{tabular}{lllll}
\hline & Incidental findings (all types) & Yes (\%) & No (\%) & Total (\%) \\
\hline & Yes (\%) & $28(7)$ & $7(2)$ & $35(9)$ \\
\hline \multirow{2}{*}{ Oncologists } & No (\%) & $69(18)$ & $279(73)$ & $348(91)$ \\
\cline { 2 - 4 } & Total (\%) & $97(25)$ & $286(75)$ & $383(100)$
\end{tabular}

2. Lungs

\section{Radiologists}

\begin{tabular}{lllll}
\hline & Incidental findings (all types) & Yes (\%) & No (\%) & Total (\%) \\
\hline \multirow{2}{*}{ Oncologists } & Yo (\%) & $14(3.7)$ & $2(0.5)$ & $16(4.2)$ \\
\cline { 2 - 4 } & Total (\%) & $56(15)$ & $311(81)$ & $367(96)$ \\
\hline
\end{tabular}

\section{If a patient had an IF in both the liver and lungs, they were included in both tables. The numbers in brackets represent the percentage of the total number of patients. IF: incidental finding \\ Interobserver agreement}

For IFs in the liver, the observed accuracy was $80 \%$, and the expected agreement was $70 \%$. The kappa value for IFs in the liver was 0.33 . The observed agreement for IFs in the lungs was $85 \%$, and the expected agreement was $79 \%$. For IFs in the lungs, the kappa was 0.28 .

\section{Sensitivities, specificities, and predictive values of incidental findings}

The oncologists' sensitivities for detecting IFs in the liver and lungs were $29 \%$ and $20 \%$, respectively. The specificities in the liver and lungs were $98 \%$ and $99 \%$, respectively. Regarding the predictive values, the 
PPVs were $80.0 \%$ in the liver and $88 \%$ in the lungs, and the corresponding NPVs were $80 \%$ and $85 \%$, respectively. The false positive rate was $20 \%$ for the liver and $12,5 \%$ for lungs.

\section{Discussions}

To our knowledge, this study is the first to investigate the interobserver variability between oncologists and radiologists when assessing the frequency of IFs on planning CT scans for postoperative radiotherapy in BC patients. The radiologists reported significantly more IFs than the oncologists (1.1 vs. 0.2 IFs per patient). This is somewhat lower that observed by Meyer et al., where 892 IFs were detected by specialized radiologists in 381 patients, which is equivalent to $2.34 \mathrm{IFs}$ per patient. Furthermore, Meyer et al. screened patient medical records for previously described abnormalities that would presumably lower the number of current IFs (8). Meyer et al detected a higher frequency of IFs compared to our study, however a significant difference between radiologists and oncologists were found.

We classified IFs into two categories: benign or potential malignant lesions. The radiologists found that $42.3 \%$ of patients had a benign finding and $24.5 \%$ had a potential malignant lesion. Previous studies have divided IFs into similar classifications, such as non-cancer-related, benign, cancer-related, or potentially important IFs $(6,8,9,12)$. Kim et al. found benign IFs in 27 of 131 scans, and 26 had cancerrelated findings (9). Ye et al. reported non-cancer-related and cancer-related IFs in 61 and 45 of 580 patients, respectively (12). Rao et al. likewise reported a low frequency of IFs in 332 patients, with 52 of patients having IFs and 31 having potentially significant findings (6). Other than the study by Meyer et al., no other studies have reported a higher percentage of IFs than the current study. The higher incidence of IFs in this study might be partly explained by the fact that the radiologists were blinded to any clinical information, and prior abnormalities were therefore not considered. Furthermore, the blinding made it difficult for the radiologists to determine whether an abnormality in the cicatrice was suspicious of recurrence of cancer or just a postoperative finding, such as inflammation or a hematoma. This may explain the high number of IFs in the breast and axilla reported by the radiologists. Kim et al. managed this by categorizing patients with postoperative changes as those with no findings (9). Thus, the majority of IFs were benign as described in previous studies, however nearly one out of four patients had a potentially malignant IF.

A statistically fair interobserver agreement was observed, with kappa values of 0.33 for the liver and 0.27 for the lungs (14). Therefore, the oncologists' findings are not purely by chance, but they are also not in perfect agreement with the radiologists' findings. However, it is unclear whether a fair agreement is clinically acceptable. Nakamura et al. also observed a difference in registration of IFs by oncologists and radiologists, where three of six patients with clinically important findings were not registered by the oncologist but only detected by the radiologist (10). This study found that the oncologists' sensitivities for detecting IFs in the liver and lungs were $29 \%$ and $20 \%$, respectively. However, access to the patients' medical records and descriptions of prior scans may have influenced the oncologists' tendency to omit IFs and thereby the sensitivity. Notably, the oncologists reported liver IFs in seven patients that the radiologists did not register, including a partial volume effect and a dilated liver vein. In the lungs, the 
oncologists only reported two IFs that the radiologists did not report. One patient had herniated fat tissue in the lung, and the other had unspecified nodules. The NPVs, representing the IFs that the oncologists did not observe, were $80 \%$ in the liver and $85 \%$ in the lungs. Even though a statistically fair agreement was found with kappa, the oncologists' low sensitivity indicates a significant difference compared to radiologists.

\section{Limitations}

Due to the retrospective design of the study, clinical follow-up was not conducted. Furthermore, the number of IFs was not adjusted for previously detected abnormalities. In addition, CT scans were reviewed by several oncologists with differing radiological qualifications and different practices regarding IFs. Finally, the quality of the planning CT scan was inferior to that of a diagnostic CT scan. In particular, the omission of the intravenous contrast agent may hinder the detection of abnormalities.

\section{Conclusions}

This study found a higher frequency of IFs reported by radiologists than that reported by oncologists on planning CT scans conducted before radiation therapy in BC patients. In terms of detecting IFs, a statistically fair interobserver agreement between the radiologists and oncologists was found. However, the oncologists detected a lower number of IFs in the liver and lungs than the radiologists. Therefore, the interobserver variability was considerable. The results of our study emphasize the need for specialized radiologists to scrutinize planning CT scans of $\mathrm{BC}$ patients prior to adjuvant radiotherapy.

\section{Abbreviations}

CT Computed tomography

IF Incidental finding

BC Breast cancer

PPV Positive predictive value

NPV Negative predictive value

\section{Declarations}

Funding: The authors declare that no funds, grants, or other support were received during the preparation of this manuscript.

Conflicts of interest: The authors have no financial or non-financial interests to disclose. 
Acknowledgements: We gratefully acknowledge the Department of Radiation Oncology at Aalborg University Hospital for their helpful contributions.

Author Contributions: All authors contributed to the study conception and design. Material preparation, data collection and analysis were performed by Stine Nyby, Signe Hertz Hansen, Agnieszka Monika Delekta and Gintare Naujokaite. The first draft of the manuscript was written by Stine Nyby and Signe Hertz, and all authors commented on previous versions of the manuscript. All authors read and approved the final manuscript

Data Availability Statement: The datasets generated during and/or analysed during the current study are available from the corresponding author on reasonable request.

Ethical approval: The study was approved by the hospital administration at AAUH as a quality assurance project.

Consent to participate: Waived.

Consent to publish: Waived.

\section{References}

1. The Danish Health Data Authority (2019) New cancer cases in Denmark.Cancer register. ;18. https://sundhedsdatastyrelsen.dk/da/tal-og-analyser/analyser-og-rapporter/sygdomme-ogbehandlinger/kraeft/kraeft-_nye-tilfaelde

2. DBCG-guideline (2020) Primary local and regional Primær lokal og regional advanced cancer mammae. Version 1.1. https://www.dmcg.dk/siteassets/kliniske-retningslinjer--skabeloner-ogvejledninger/kliniske-retningslinjer-opdelt-pa-dmcg/dbcg/dbcg_labc-v-1.1_admgodk291020.pdf? fbclid=IwAR2SQL2fynggMpNSOvEXuKIHsmZIDsFugUWik4R_t9NvgsuVvIO62uDQEkM. Accessed 13. december 2021

3. Cardoso F, Kyriakides S, Ohno S, Penault-Llorca F, Poortmans P, Rubio IT, Zackrisson S, Senkus E (2019) Early breast cancer: ESMO Clinical Practice Guidelines for diagnosis, treatment and followupt. Ann Oncol 30:1194-1220. https://doi.org/10.1093/annonc/mdz173

4. DBCG-guideline (2019) Surgical breast cancer treatment. Version 2.0. https://www.dmcg.dk/siteassets/kliniske-retningslinjer-skabeloner-og-vejledninger/kliniskeretningslinjer-opdelt-pa-dmcg/dbcg/dbcg_kirurgisk-behandling_v2.0_adm.godk_250321.pdf? fbclid=IwAR3oxG165dNKdbYt5Dumb-AR8qXxeWaDXI-VfLnW1qvb-gc9YzC-TD8wwRs. Accessed 13. december 2021

5. DBCG-Guideline (2021) Postoperative radiotherapy treatment of breast cancer. Version 2.0. https://www.dmcg.dk/siteassets/kliniske-retningslinjer-skabeloner-og-vejledninger/kliniskeretningslinjer-opdelt-pa-dmcg/dbcg/dbcg_postop_stralebeh_v2_0_admgodk250121.pdf? 
fbclid=IwAR3pWjFqEIBpHS9fmkxAaen4ywCuCvtZCZTw6PLSP5KfJV5GK60rupjNEDw. Accessed 13. december 2021

6. Rao S, Patel A, Levin K, Lu M, Garbarino K, Myers D, Walker EM, Ryu S, Ho Kim J, Movsas B (2010) How often are previously undetected radiographic abnormalities detected at the time of CT simulation for breast cancer patients. Am J Clin Oncol 33:262-264.

https://doi.org/10.1097/COC.0b013e3181a76a3d

7. Orme NM, Fletcher JG, Siddiki HA, Harmsen WS, O'Byrne MM, Port JD, Tremaine WJ, Pitot HC, McFarland EG, Robinson ME, Koenig BA, King BF, Wolf SM (2010) Incidental Findings in Imaging Research. Arch Intern Med 170(17):1525-1532. https://doi:10.1001/archinternmed.2010.317

8. Meyer HJ, Uhlig J, Schramm D, Bach AG, Surov A (2017) Radiological findings on irradiation-CT in patients with non metastatic breast cancer. $\mathrm{Br} \mathrm{J}$ Radiol 90:20170162. https://doi.org/10.1259/bjr.20170162

9. Kim MJ, Kim EK, Keum KC, Park BW, Son EJ, Oh KK (2008) Treatment-planning CT scan for breast and chest-wall irradiation: how many unexpected abnormalities could we detect. Clin Imaging 32:443-446. https://doi.org/10.1016/j.clinimag.2008.02.025

10. Nakamura N, Tsunoda H, Takahashi O, Kikuchi M, Honda S, Shikama N, Akahane K, Sekiguchi K (2012) Frequency and clinical significance of previously undetected incidental findings detected on computed tomography simulation scans for breast cancer patients. Int J Radiat Oncol Biol Phys 84:602-605. https://doi.org/10.1016/j.jijobp.2011.12.075

11. Smitt MC, Mehta VK (2001) Is diagnostic review of radiotherapy-planning CT scans important in the conformal therapy era. AJR Am J Roentgenol 177:521-524.

https://doi.org/10.2214/ajr.177.3.1770521

12. Ye JC, Truong MT, Kachnic LA, Subramaniam RM, Hirsch AE (2011) Implications of previously undetected incidental findings on 3D CT simulation scans for radiation therapy. Pract Radiat Oncol 1:22-26. https://doi.org/10.1016/j.prro.2010.09.001

13. Edge SB, Compton CC (2010) The American Joint Committee on Cancer: the 7th edition of the AJCC cancer staging manual and the future of TNM. Ann Surg Oncol 17:1471-1474. https://doi.org/10.1245/s10434-010-0985-4

14. Viera AJ, Garrett JM (2005) Understanding interobserver agreement: the kappa statistic. Fam Med $37: 360-363$

\section{Figures}




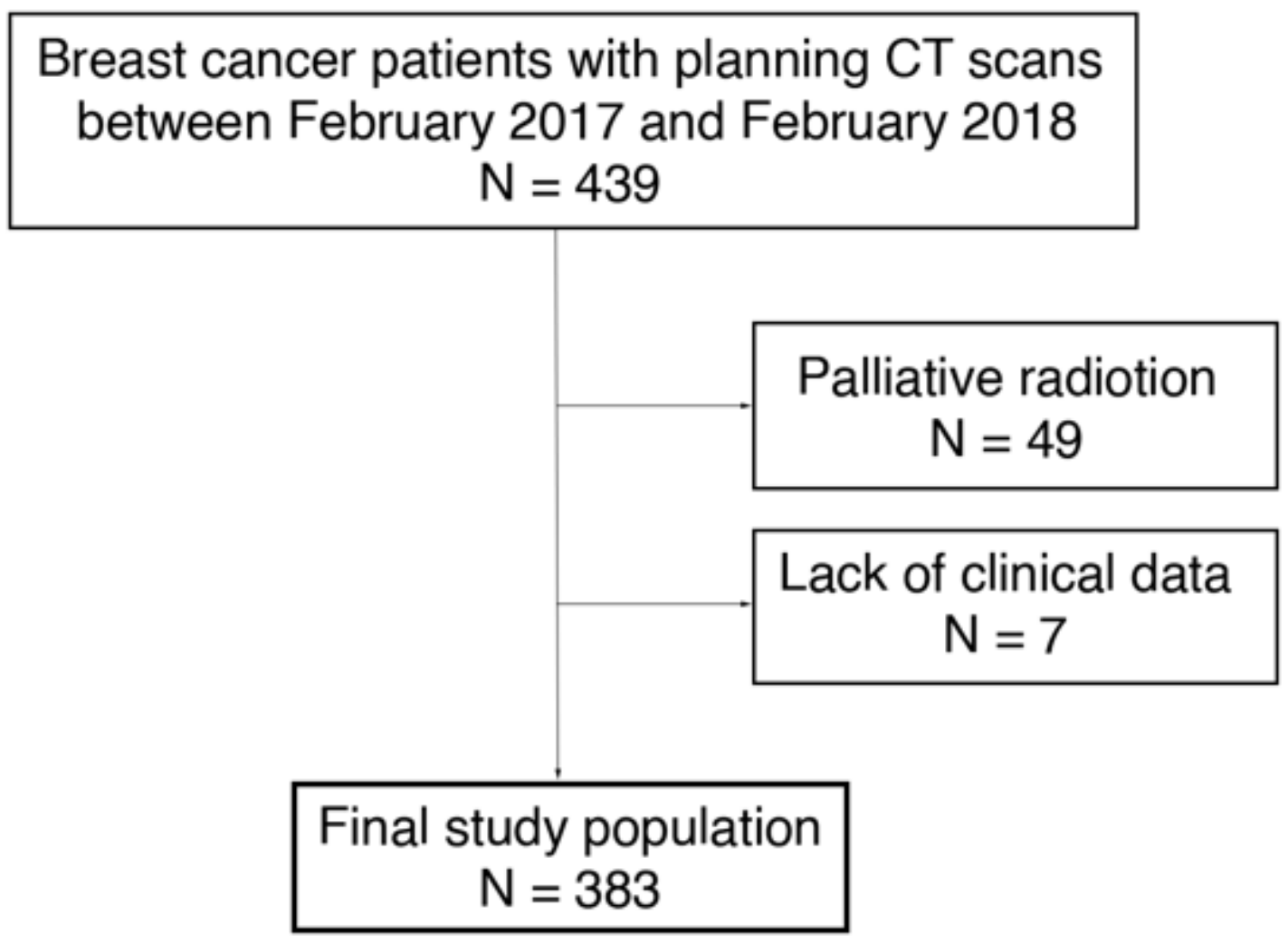

Figure 1

Flowchart of the 383 breast cancer patients with a planning CT scan between February 2017 and February 2018

CT: computed tomography 